\title{
Cooperative Sensor Networks with Misinformed Sensors
}

\author{
Zhiyu Yang and Lang Tong \\ School of Electrical and Computer Engineering \\ Cornell University, Ithaca, NY 14853, USA \\ Email: $\{z y 26$, ltong $\} @$ ece.cornell.edu
}

\begin{abstract}
We consider the communication from a cooperative sensor network to a mobile access point. We assume that sensors are informed with a global message and some nodes are misinformed with random messages. Nodes transmit to the mobile access point in a pre-scheduled order. We derive an achievable rate for the information retrieval process when $d$ sensors are transmitting at a time. For the Gaussian multiple access channel under the total network power constraint, we show that the maximum achievable rate for the Gaussian multiple access channel is $O\left(\log _{2} d\right)$, obeying the same scaling law as the capacity of an Gaussian multiple-input-single-output channel.
\end{abstract}

\section{INTRODUCTION}

We consider information retrieval in a cooperative SEnsor Network with Mobile Access (SENMA) [1]. As illustrated in Fig. 1, SENMA contains two types of nodes: a large number of low power geographically distributed sensors, and a computationally powerful mobile access point. By cooperative SENMA (C-SENMA) we mean that, in communicating to the mobile access point, sensors may reach an agreement on the message to transmit, and appropriate coding can be implemented across sensors. This makes the information retrieval robust against failure of individual sensors.

If no sensor is misinformed, and if the mobile access point polls one sensor at a time and the channel between each sensor and the mobile access point is a discrete memoryless channel $q(y \mid x)$, then the maximum achievable rate for the information retrieval is given by

$$
C_{0}=\max _{p(x)} I(X ; Y)
$$

In such a setting, there is no difference between retrieving information from a single sensor or multiple sensors since all sensors have the same message.

For large scale sensor networks, however, reaching complete agreement among all sensors is very difficult, if not impossible. For example, a software agent responsible for distributing the message may not have reached all sensors, or sensors make errors due to unreliable conference links. In practice, there is always a possibility that some sensors do not have the correct message for transmission. We refer to such sensors as misinformed. The achievable rate of cooperative sensor networks with misinformed sensors is no longer obvious.

In this paper, we investigate the achievable rate for multiple simultaneous transmission (allowing $d$ nodes to transmit at a time). An achievable rate is given in Theorem 2. We then

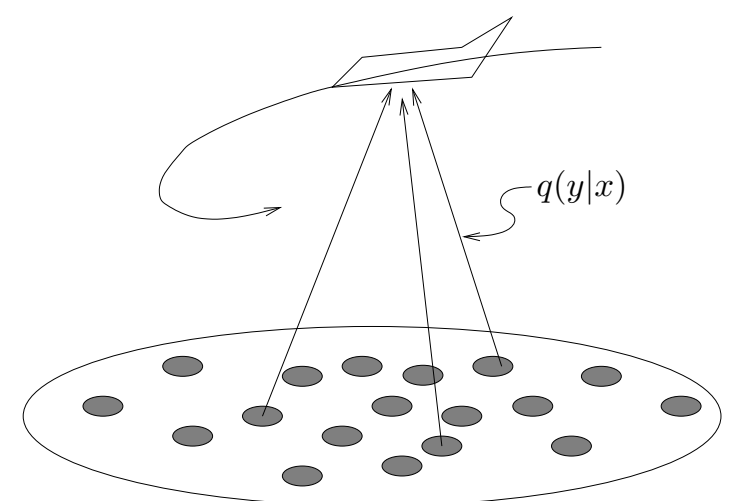

Fig. 1. SEnsor Network with Mobile Access

consider an additive Gaussian multiple access channel with a total power constraint and show that the maximum achievable rate is $O\left(\log _{2} d\right)$, obeying the same rule as the case when there is no misinformed sensors.

For notation compactness, denote an entry with two subindices $i$ and $j$ by $(\cdot)_{i j}$. The meaning of $i j$, a double-index or a scalar multiplication, can be determined by its context.

\section{MODEL}

The communication of the global message from the network to the mobile access point is divided into four steps as shown in Fig. 2: (a) orientation, (b) scheduling, (c) transmission and reception, and (d) decoding. In the first step, nodes are informed with the globe message $W \in\{1, \ldots, M\}$ that is uniformly distributed. Due to the size of the network, a node may be informed incorrectly and end up with a different message. We assume that each node receives the globe message correctly with some probability, and the reception is independent of other nodes. More specifically, the reception of node $i$ is controlled by a binary random variable $U_{i}$, independent of $W$ and identically independently distributed (i.i.d.) across node index $i$ with distribution

$$
p\left(u_{i}\right)= \begin{cases}\beta & \text { if } u_{i}=0 \\ 1-\beta & \text { if } u_{i}=1\end{cases}
$$

where $\beta \in[0,1]$ is a constant. When $U_{i}=1$, the received message at node $i, \widetilde{W}_{i}$, is equal to the globel message $W$. 


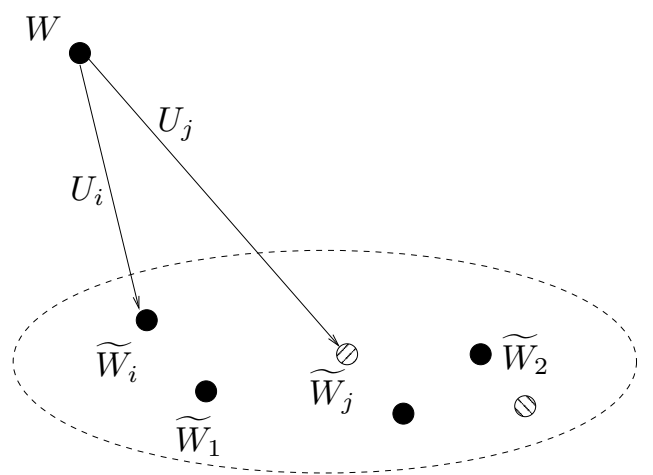

(a) Step I: Orientation at $t=0$.

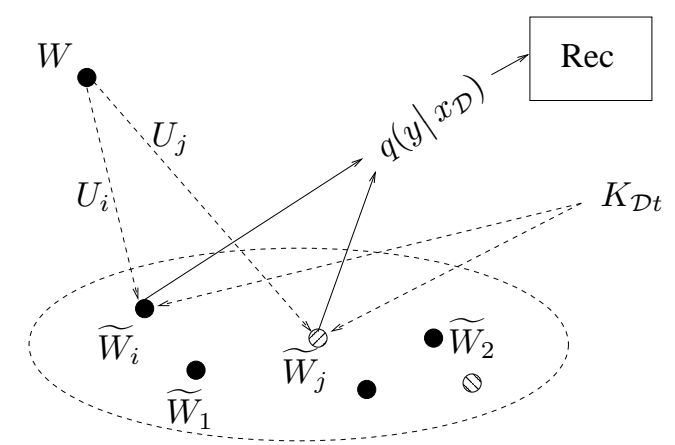

(c) Step III: Transmission and Reception at $t=$ $1, \ldots, n$.

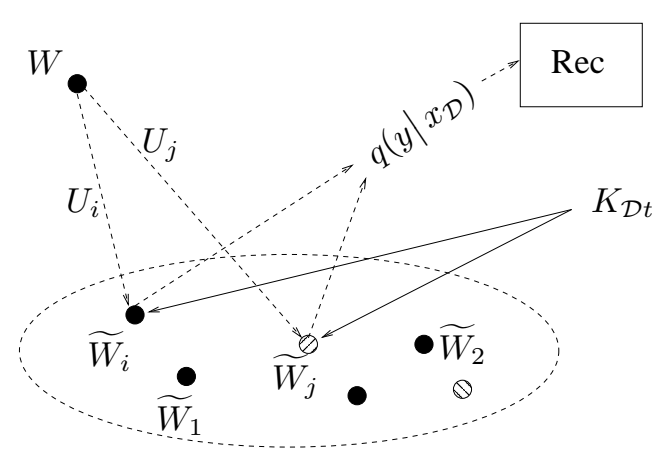

(b) Step II: Scheduling at $t=1, \ldots, n$. Here $i, j \in k_{\mathcal{D} t}$.

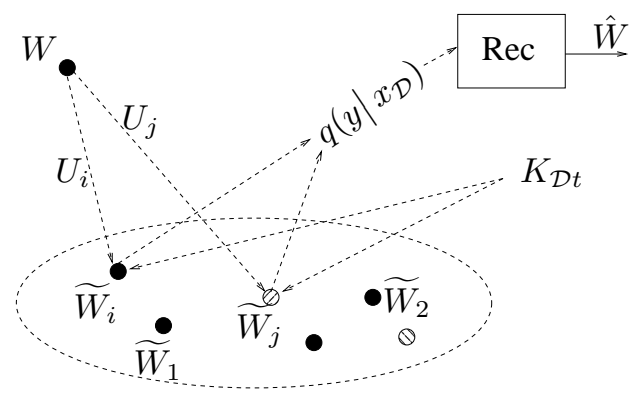

(d) Step IV: Decoding at $t=n$.

Fig. 2. Communication steps.

When $U_{i}=0, \widetilde{W}_{i}$ is uniformly distributed from 1 to $M$. Thus

$$
p\left(\tilde{w}_{i} \mid w, u_{i}\right)= \begin{cases}\delta\left(\tilde{w}_{i}, w\right) & \text { if } u_{i}=1 \\ \frac{1}{M} 1_{1 \leq \tilde{w}_{i} \leq M} & \text { if } u_{i}=0\end{cases}
$$

where $\delta(a, b)$ is equal to 1 if $a=b, 0$ otherwise, and the indicating function $1_{A}$ equal to 1 if event $A$ is true, 0 otherwise. The constant $\beta$ controls the reception of the globe message by individual nodes and is referred to as the orientation error probability of the network.

The mobile access point comes to retrieve information from the field after the information orientation has been accomplished. Let $\mathcal{D} \triangleq(1, \ldots, d)$ and $Z_{\mathcal{D}}$ denote $\left(Z_{1}, \ldots, Z_{d}\right)$, where $Z_{1}, \ldots, Z_{d}$ are generic symbols. We assume that $d$ nodes are scheduled to transmit at each time slot, each transmitting one symbol to the channel Denote $K_{\mathcal{D} t}=$ $\left(K_{1 t}, \ldots, K_{d t}\right)$ the nodes transmitting at time $t$. The scheduling signal $K_{\mathcal{D} t}$ is preset before the deployment of the sensors, thus it does not adapt to the mobile access point's reception.

We assume that the uplink multiple access channels (MACs) from any $d$ nodes to the mobile access point are identical and modeled by a discrete memoryless $\operatorname{MAC}\left\{\mathcal{X}, \mathcal{Y}, q\left(y \mid x_{\mathcal{D}}\right)\right\}$, where $\mathcal{X}$ and $\mathcal{Y}$ are the input and output alphabets respectively, and $q\left(y \mid x_{\mathcal{D}}\right)$ is the transition probability of the channel. We assume the MAC is symmetrical with respect to input permutations, i.e., $q\left(y \mid x_{\pi_{1}}, \ldots, x_{\pi_{d}}\right)$ is identical for all permutations $\pi_{\mathcal{D}}$ in the domain $\mathcal{D}$. Denote $X_{j t}$ the transmission from node $K_{j t}$ and $Y_{t}$ the output of the MAC at time $t$. The total number of slots of transmission is $n$, the number of time slots the mobile access point spends to retrieve information from the field.

In the last step, the access point decodes the globe message based on the observation of $Y^{n}$ and the scheduling $K_{\mathcal{D}}^{n}$. The decoded message is denoted by $\hat{W} \in\{1, \ldots, M\}$. Let $W_{j t} \triangleq$ $\widetilde{W}_{K_{j t}}$ be the message at the $j t$-th polled node. We assume that the sensor network is large in the sense that there are infinite number of nodes, and there is no limit on how many times a node can transmit. The large network assumption is to make sure that with zero probability that all nodes have the incorrect message, thus it is possible to have non-zero achievable rate.

Let $\mathcal{N}$ be the set of positive numbers and $\mathcal{W} \triangleq$ $\{1,2, \ldots, M\}$. The rate of a code book is defined as $R \triangleq$ $\log (M) / n$, where $M$ is the number of messages in the code book and $n$ is the length of a codeword. The probability of error is defined as $\mathcal{P}_{e} \triangleq \mathcal{P}(\hat{W} \neq W)$, where $W \in \mathcal{W}$ is uniformly distributed and $\hat{W}$ is the decoded message. A rate $R$ is called achievable if for any given error $\epsilon>0$, there exists a code book with rate large than $R-\epsilon$ and probability of error less than $\epsilon$.

\section{AChiEvable RATE For $d$ Simultaneous TRANSMISSIONS}

As a special case when $d=1$, the maximum achievable rate is given by the following theorem: 
Theorem 1: For $d=1$, the maximum achievable rate of a C-SENMA $(\beta, \mathcal{X}, \mathcal{Y}, q(y \mid x))$ is

$$
C_{1}=(1-\beta) \max _{p(x)} I(X ; Y),
$$

where $X \in \mathcal{X}, Y \in \mathcal{Y}$, and $p(y \mid x)=q(y \mid x)$.

For $d>1$, an achievable rate is given as follows:

Theorem 2: For a given $d$, consider random variables $X_{\mathcal{D}}$ with distribution $p\left(x_{\mathcal{D}}\right)$. Denote $p_{\mathcal{I}}\left(x_{\mathcal{I}}\right)$ the marginal distribution of $X_{\mathcal{I}}$ for $\mathcal{I} \subset \mathcal{D}$. The following rate is achievable for C-SENMA $\left(\beta, \mathcal{X}, \mathcal{Y}, q\left(y \mid x_{\mathcal{D}}\right)\right)$,

$$
C_{d}=\max _{p\left(x_{\mathcal{D}}\right)} \sum_{\mathcal{I} \subset \mathcal{D}}(1-\beta)^{|\mathcal{I}|} \beta^{d-|\mathcal{I}|} I\left(X_{\mathcal{I}}^{(\mathcal{I})} ; Y\right),
$$

where $X_{\mathcal{D}}^{(\mathcal{I})}=\left(X_{1}^{(\mathcal{I})}, \ldots, X_{d}^{(\mathcal{I})}\right)$ are derived from $X_{\mathcal{D}}$ with distribution

$$
p^{(\mathcal{I})}\left(x_{\mathcal{D}}^{(\mathcal{I})}\right)=p_{\mathcal{I}}\left(x_{\mathcal{I}}^{(\mathcal{I})}\right) \prod_{j \notin \mathcal{I}} p_{j}\left(x_{j}^{(\mathcal{I})}\right)
$$

and $p\left(y \mid x_{\mathcal{D}}^{(\mathcal{I})}\right)=q\left(y \mid x_{\mathcal{D}}^{(\mathcal{I})}\right)$.

The achievability of Theorem 2 is outlined in the appendix

\section{Gaussian Multiple Access Channels with a TOTAL POWER CONSTRAINT}

Consider the following Gaussian multiple access channel

$$
y=v+\sum_{i} x_{i}
$$

where $x_{i} \in \mathcal{C}$ is the input from the $i$-th sensor, $v \in \mathcal{C}$ is the additive white Gaussian noise with zero mean and unit variance, and $y \in \mathcal{C}$ is the channel output. We impose a total power constraint $P$ on the network, i.e., the total transmitted power from all sensors is less than or equal to $P$ :

$$
\frac{1}{n} \sum_{t=1}^{n} \sum_{j=1}^{d}\left|x_{j t}\right|^{2} \leq P
$$

where $x_{j t}$ is the $j t$-th transmission. If there is no orientation error, i.e., $\beta=0$, we know from the multiple-input-singleoutput (MISO) channel capacity that, with $d$ sensor polled at a time, the maximum achievable rate is $C_{d}^{(0)}=\log _{2}(1+d P)$. Therefore, when $\beta=0$, the maximum achievable rate $C_{d}^{(0)}=$ $O\left(\log _{2} d\right)$ goes to infinity as we increase $d$, the number of sensors polled at a time. In this section, we show that even with $\beta>0$, the maximum achievable rate of the channel (1) is still $O\left(\log _{2} d\right)$.

Consider activating $d$ sensors at a time. Let the input random variables $X_{1}, \ldots, X_{d}$ be identically Gaussian distributed $\mathcal{N}_{C}(0, P / d)$ and let any two input random variables $X_{i}, X_{j}$ have correlation coefficient 1 . For $\mathcal{I} \subset \mathcal{D}$, since the derived random variables $X_{\mathcal{D} \backslash \mathcal{I}}^{(\mathcal{I})}$, independent of each other, are independent of $X_{\mathcal{I}}^{(\mathcal{I})}, X_{\mathcal{D} \backslash \mathcal{I}}^{(\mathcal{I})}$ contribute $(d-|\mathcal{I}|) P / d$ power to the additive noise. Therefore,

$$
I\left(X_{\mathcal{I}}^{(\mathcal{I})} ; Y\right)=\log _{2}\left(1+\frac{|\mathcal{I}|^{2} P / d}{1+(d-|\mathcal{I}|) P / d}\right) .
$$

From Theorem 2, the following rate is achievable,

$$
R_{d}=\sum_{i=0}^{d}(1-\beta)^{i} \beta^{d-i}\left(\begin{array}{l}
d \\
i
\end{array}\right) \log _{2}\left(1+\frac{i^{2} P / d}{1+(d-i) P / d}\right) .
$$

The next proposition shows that $R_{d}=O\left(\log _{2} d\right)$. Since $R_{d} \leq$ $C_{d} \leq C_{d}^{(0)}=O\left(\log _{2} d\right)$, we have $C_{d}=O\left(\log _{2} d\right)$.

Proposition 3: For $\beta \in[0,1)$ and $P>0$,

$$
\lim _{d \rightarrow \infty}\left(R_{d}-\log _{2} d\right)=\log _{2}\left(\frac{(1-\beta)^{2} P}{1+\beta P}\right) .
$$

Proof: Let $S_{1}, S_{2}, \ldots$, be i.i.d. Bernoulli with mean $1-$ $\beta$. Then $T_{d}=\sum_{i=1}^{d} S_{i}$ is binomial distributed. Let

$$
f(a, b) \triangleq \log _{2}\left(a+\frac{b^{2} P}{1+(1-b) P}\right) .
$$

We have

$$
\begin{aligned}
R_{d}-\log _{2} d & =\sum_{i=0}^{d}(1-\beta)^{i} \beta^{d-i}\left(\begin{array}{l}
d \\
i
\end{array}\right) f(1 / d, i / d) \\
& =E\left[f\left(1 / d, T_{d} / d\right)\right] .
\end{aligned}
$$

Since $f(a, b)$ is continues at $(0,1-\beta)$, for all $\epsilon>0$, there exists a $\delta>0$ such that for all $a \in[0, \delta) \triangleq \mathcal{A}$ and $b \in$ $(1-\beta-\delta, 1-\beta+\delta) \triangleq \mathcal{B},|f(a, b)-f(0,1-\beta)| \leq \epsilon / 3$. For the same $\epsilon$ and $\delta$,

$$
\begin{aligned}
&\left|E\left[f\left(1 / d, Y_{d} / d\right)\right]-f(0,1-\beta)\right| \\
& \leq E\left[\left|f\left(1 / d, Y_{d} / d\right)-f(0,1-\beta)\right|\right] \\
& \leq E_{Y_{d} / d \in \mathcal{B}}\left[\left|f\left(1 / d, Y_{d} / d\right)-f(0,1-\beta)\right|\right] \\
&+E_{Y_{d} / d \notin \mathcal{B}}\left[\left|f\left(1 / d, Y_{d} / d\right)\right|\right] \\
&+\mathcal{P}_{r}\left(Y_{d} / d \notin \mathcal{B}\right) f(0,1-\beta) .
\end{aligned}
$$

For large $d, 1 / d \in \mathcal{A}$. Therefore, due to the continuity of $f$, the first term in (2) is upper bounded by $\epsilon / 3$. The third term, by the weak law of large number, is upper bounded by $\epsilon / 3$ for large $d$. We bound the second term as follows. For $d \geq 1$ and $i=0, \ldots, d$, we have

$$
\begin{aligned}
& f(1 / d, i / d) \leq \log _{2}(1 / d+P), \\
& f(1 / d, i / d) \geq \log _{2}(1 / d) .
\end{aligned}
$$

For large $d$ such that $1 / d+P \leq d$, we have

$$
|f(1 / d, i / d)| \leq \log _{2} d
$$

Hence, by Chebyshev inequality,

$$
\begin{aligned}
E_{Y_{d} / d \notin \mathcal{B}}\left[\left|f\left(1 / d, Y_{d} / d\right)\right|\right] & \leq \mathcal{P}_{r}\left(Y_{d} / d \notin \mathcal{B}\right) \log _{2} d \\
& \leq \frac{\sigma_{s}^{2}}{\delta^{2} d} \log _{2} d \\
& \leq \epsilon / 3 \quad \text { for large } d
\end{aligned}
$$

Therefore, $\left|E\left[f\left(1 / d, Y_{d} / d\right)\right]-f(0,1-\beta)\right| \leq \epsilon$ for large $d$. Since $\epsilon>0$ is arbitrary, $\lim _{d \rightarrow \infty} E\left[f\left(1 / d, Y_{d} / d\right)\right]=f(0,1-$ $\beta)$, proving the proposition.

Fig. 3 shows the achievable rate $R_{d}$ and the approximation function $\log _{2} d+f(0,1-\beta)$ versus $d$ when $P=10$ and $\beta=$ 


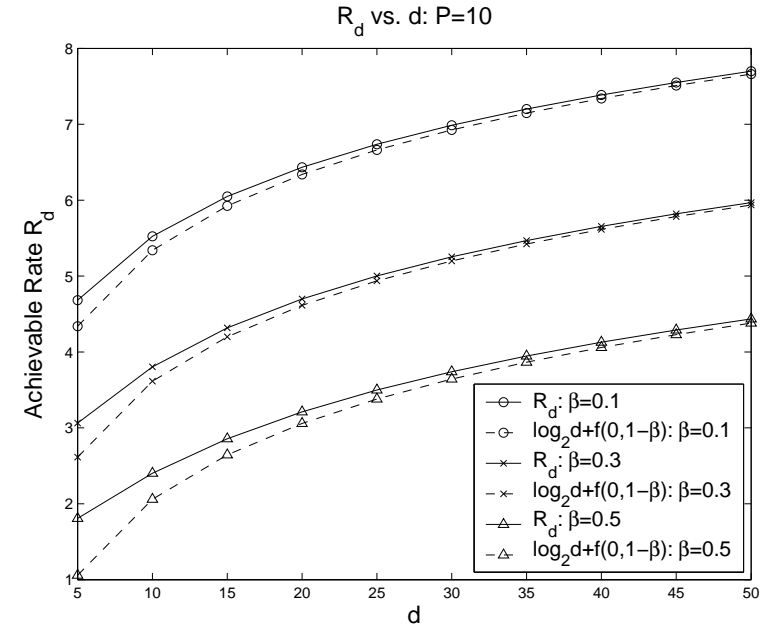

Fig. 3. Achievable rate $R_{d}$ versus $d: P=10$ and $\beta=0.1,0.3,0.5$.

0.1, 0.3, 0.5. As shown in Fig. 3, $R_{d}$ and the approximation function converge as $d$ increases. As expected, the achievable rate is higher for a network with a smaller orientation error probability.

\section{SUMMARY}

We have presented an achievable rate for cooperative sensor networks with misinformed sensors when $d$ sensors are activated at a time. We have considered an additive Gaussian multiple access channel with a network power constraint and shown that the maximum achievable rate is $O\left(\log _{2} d\right)$.

\section{APPENDIX}

\section{SKETCH OF THE ACHIEVABILITY}

We first derive an achievable rate based on a repetitive scheduling, and then optimize the achievable rate to proof the achievability of Theorem 2 .

We group every $N$ time slots into a time frame and schedule in a time frame $d$ nodes that have never be scheduled before. This scheme is called $N$-scheduling.

Lemma 4: For a given $d$, consider random variables $S_{\mathcal{D}} \in$ $\mathcal{X}^{d}$ with distribution $p\left(s_{\mathcal{D}}\right)$. Denote $p_{\mathcal{I}}\left(s_{\mathcal{I}}\right)$ the marginal distribution of $S_{\mathcal{I}}$ for $\mathcal{I} \subset \mathcal{D}$. Let $\overline{\mathcal{I}} \triangleq \mathcal{D} \backslash \mathcal{I}$. For a given $\mathrm{C}$ SENMA $\left(\beta, \mathcal{X}, \mathcal{Y}, q\left(y \mid x_{\mathcal{D}}\right)\right)$, with the 1 -scheduling, the rate

$$
R_{d 1} \triangleq I\left(S_{\mathcal{D}} ; Y\right)
$$

is achievable, where

$p\left(y \mid s_{\mathcal{D}}\right)=\sum_{\mathcal{I} \subset \mathcal{D}}(1-\beta)^{|\mathcal{I}|} \beta^{|\overline{\mathcal{I}}|} \sum_{s_{\overline{\mathcal{I}} \in \mathcal{X}|\overline{\mathcal{I}}|}} q\left(y \mid s_{\mathcal{I}}, s_{\overline{\mathcal{I}}}^{\prime}\right) \prod_{j \in \overline{\mathcal{I}}} p_{j}\left(s_{j}^{\prime}\right)$.

Proof: Code book Generation: Generate a code book with $M=2^{n R}$ messages at random according to the distribution $p\left(s_{\mathcal{D}}\right)$. Specifically, for $1 \leq t \leq n$, let $s_{\mathcal{D} t}(w)=$ $\left(s_{1 t}(w), \ldots, s_{d t}(w)\right)$ be a mapping from $\mathcal{W}$ to $\mathcal{X}^{d}$. For $1 \leq$ $t \leq n, 1 \leq w \leq M$, assign $s_{\mathcal{D} t}(w)$ a value independently generated according to the distribution $p\left(s_{\mathcal{D}}\right)$. After the random assignment of $s_{\mathcal{D} t}(w)$, let every node have the same code book. At time $t$, the node $k_{i t}, 1 \leq i \leq d$, transmits symbol $s_{i t}\left(\tilde{w}_{k_{i t}}\right)$ to the channel.

Decoder: Typical set decoding is employed. Define the typical set $A_{\epsilon}^{(n)}$ with respect to the distribution $p\left(s_{\mathcal{D}}, y\right)$

$$
\begin{aligned}
A_{\epsilon}^{(n) \triangleq} & \left\{\left(s_{\mathcal{D}}^{n}, y^{n}\right) \in \mathcal{X}^{d n} \times \mathcal{Y}^{n}:\right. \\
& \left|-\frac{1}{n} \log p\left(s_{\mathcal{D}}^{n}\right)-H\left(S_{\mathcal{D}}\right)\right| \leq \epsilon, \\
& \left|-\frac{1}{n} \log p\left(y^{n}\right)-H(Y)\right| \leq \epsilon, \\
& \left.\left|-\frac{1}{n} \log p\left(s_{\mathcal{D}}^{n}, y^{n}\right)-H\left(S_{\mathcal{D}}, Y\right)\right| \leq \epsilon\right\},
\end{aligned}
$$

where $p\left(s_{\mathcal{D}}^{n}, y^{n}\right)=\prod_{i=1}^{n} p\left(s_{\mathcal{D} i}, y_{i}\right)$. Upon receiving channel outputs $y^{n}$, the mobile access point declares the message $\hat{w}$ as the received message if there is one and only one $\hat{w} \in \mathcal{W}$ such that $\left(s_{\mathcal{D}}^{n}(\hat{w}), y^{n}\right) \in A_{\epsilon}^{(n)}$; otherwise, the decoder declares an error.

The error analysis is omitted here due to the space limit. It can be shown that, if $R<I\left(S_{\mathcal{D}} ; Y\right)$, the average probability of error, average over all codewords and all code books, converges to zero as $n$ goes to infinity.

Corollary 5: For a given $d$, a distribution $p\left(s_{\mathcal{D}}\right)$ on the alphabet $\mathcal{X}^{d}$, and a C-SENMA $\left(\beta, \mathcal{X}, \mathcal{Y}, q\left(y \mid x_{\mathcal{D}}\right)\right)$, with the $N$-scheduling, the rate

$$
R_{d N} \triangleq \frac{1}{N} I\left(S_{\mathcal{D}}^{N} ; Y^{N}\right)
$$

is achievable, where $S_{\mathcal{D}}^{N} \in \mathcal{X}^{d N}, Y^{N} \in \mathcal{Y}^{N}$, and

$$
\begin{aligned}
p\left(s_{\mathcal{D}}^{N}, y^{N}\right)= & \left(\prod_{i=1}^{N} p\left(s_{\mathcal{D} i}\right)\right)\left(\sum_{\mathcal{I} \subset \mathcal{D}}(1-\beta)^{|\mathcal{I}|} \beta^{|\overline{\mathcal{I}}|}\right. \\
& \left.\cdot \prod_{i=1}^{N} \sum_{s_{\overline{\mathcal{I}} \in \mathcal{X}|\overline{\mathcal{I}}|}} q\left(y_{i} \mid s_{\mathcal{I} i}, s_{\overline{\mathcal{I}}}^{\prime}\right) \prod_{j \in \overline{\mathcal{I}}} p_{j}\left(s_{j}^{\prime}\right)\right) .
\end{aligned}
$$

Proof: Consider the $N$-th extension of the MAC, $\left(\mathcal{X}^{N}, \mathcal{Y}^{N}, q\left(y^{N} \mid x_{\mathcal{D}}^{N}\right)\right)$, where

$$
q\left(y^{N} \mid x_{\mathcal{D}}^{N}\right)=\prod_{i=1}^{N} q\left(y_{i} \mid x_{\mathcal{D} i}\right)
$$

Let $p\left(s_{\mathcal{D}}^{N}\right)=\prod_{i=1}^{N} p\left(s_{\mathcal{D} i}\right)$ be the input distribution to the $N$-th extended C-SENMA $\left(\beta, \mathcal{X}^{N}, \mathcal{Y}^{N}, q\left(y^{N} \mid x_{\mathcal{D}}^{N}\right)\right)$. By Lemma 4, $I\left(S_{\mathcal{D}}^{N} ; Y^{N}\right)$ is achievable for the $N$-th extended system with 1-scheduling, where

$$
\begin{aligned}
p\left(s_{\mathcal{D}}^{N}, y^{N}\right)= & \left(\prod_{i=1}^{N} p\left(s_{\mathcal{D} i}\right)\right)\left(\sum_{\mathcal{I} \subset \mathcal{D}}(1-\beta)^{|\mathcal{I}|} \beta^{|\overline{\mathcal{I}}|}\right. \\
& \left.\cdot \sum_{s_{\overline{\mathcal{I}}}^{N} \in \mathcal{X}^{N|\overline{\mathcal{I}}|}} \prod_{i=1}^{N} q\left(y_{i} \mid s_{\mathcal{I} i}, s_{\overline{\mathcal{I}}_{i}}^{\prime}\right) \prod_{j \in \overline{\mathcal{I}}} p_{j}\left(s_{j i}^{\prime}\right)\right) \\
= & \left(\prod_{i=1}^{N} p\left(s_{\mathcal{D} i}\right)\right)\left(\sum_{\mathcal{I} \subset \mathcal{D}}(1-\beta)^{|\mathcal{I}|} \beta^{|\overline{\mathcal{I}}|}\right. \\
& \left.\cdot \prod_{i=1}^{N} \sum_{s_{\overline{\mathcal{I}} \in \mathcal{X}|\overline{\mathcal{I}}|}} q\left(y_{i} \mid s_{\mathcal{I} i}, s_{\overline{\mathcal{I}}}^{\prime}\right) \prod_{j \in \overline{\mathcal{I}}} p_{j}\left(s_{j}^{\prime}\right)\right) .
\end{aligned}
$$


The operation of the original C-SENMA with the $N$ scheduling is equivalent to that of the $N$-th extended CSENMA with the 1-scheduling. Thus $\frac{1}{N} I\left(S_{\mathcal{D}}^{N} ; Y^{N}\right)$ is achievable for the original system with $N$-scheduling.

We are now ready to prove the achievability of Theorem 2 by showing the convergence of $R_{d N}$ as $N$ goes to infinity. Let $S_{\mathcal{D}}^{N}$ has distribution $\prod_{i=1}^{N} p\left(s_{\mathcal{D} i}\right)$. Introduce $V_{\mathcal{D}} \in\{0,1\}^{d}$, i.i.d. Bernoulli random variables with mean $1-\beta$. Let $V_{\mathcal{D}}$ be independent of $S_{\mathcal{D}}^{N}$. It can be shown that if we let

$$
p\left(y^{N} \mid s_{\mathcal{D}}^{N}, v_{\mathcal{D}}\right)=\prod_{i=1}^{N} \sum_{s_{\overline{\mathcal{I}}}^{\prime} \in \mathcal{X}|\overline{\mathcal{I}}|} q\left(y_{i} \mid s_{\mathcal{I} i}, s_{\overline{\mathcal{I}}}^{\prime}\right) \prod_{j \in \overline{\mathcal{I}}} p_{j}\left(s_{j}^{\prime}\right)
$$

where $\mathcal{I}=\left\{i: v_{i}=1\right\}$, then the resulting marginal distribution $p\left(s_{\mathcal{D}}^{N}, y^{N}\right)$ is given by (3). Therefore,

$$
\begin{aligned}
\frac{1}{N} I\left(S_{\mathcal{D}}^{N} ; Y^{N}\right) & =\frac{1}{N} I\left(S_{\mathcal{D}}^{N} ; Y^{N}, V_{\mathcal{D}}\right)-\frac{1}{N} I\left(S_{\mathcal{D}}^{N} ; V_{\mathcal{D}} \mid Y^{N}\right) \\
& \geq \frac{1}{N} I\left(S_{\mathcal{D}}^{N} ; Y^{N} \mid V_{\mathcal{D}}\right)-\frac{d}{N} \\
& \rightarrow \frac{1}{N} I\left(S_{\mathcal{D}}^{N} ; Y^{N} \mid V_{\mathcal{D}}\right) \quad \text { as } N \rightarrow \infty
\end{aligned}
$$

where (5) is because $H\left(V_{\mathcal{D}}\right) \leq d$. For all $v_{\mathcal{D}} \in\{0,1\}^{d}$, let $\mathcal{I}=\left\{i: v_{i}=1\right\}$. Let $X_{\mathcal{D}}^{(\mathcal{I})}$, derived from $p\left(s_{\mathcal{D}}\right)$, and $\mathcal{Y}$ be defined as in Theorem 2. It can be shown from (4) that

$$
\frac{1}{N} I\left(S_{\mathcal{D}}^{N} ; Y^{N} \mid v_{\mathcal{D}}\right)=I\left(X_{\mathcal{I}}^{(\mathcal{I})} ; Y\right) \text {. }
$$

Hence,

$$
\begin{aligned}
\lim _{N \rightarrow \infty} R_{d N} & =\lim _{N \rightarrow \infty} \frac{1}{N} I\left(S_{\mathcal{D}}^{N} ; Y^{N}\right) \\
& \geq \sum_{\mathcal{I} \subset \mathcal{D}}(1-\beta)^{|\mathcal{I}|} \beta^{d-|\mathcal{I}|} I\left(X_{\mathcal{I}}^{(\mathcal{I})} ; Y\right) .
\end{aligned}
$$

Optimizing over $p\left(s_{\mathcal{D}}\right)$ concludes the proof of the achievability of Theorem 2.

\section{ACKNOWLEDGMENT}

This work was supported in part by the Multidisciplinary University Research Initiative (MURI) under the Office of Naval Research Contract N00014-00-1-0564 and the National Science Foundation under Contract CCR-0311055.

\section{REFERENCES}

[1] L. Tong, Q. Zhao, and S. Adireddy, "Sensor Networks with Mobile Agents," in Proc. 2003 Military Communications Intl Symp., Boston, MA, Oct. 2003. 